\title{
柴达木盆地水生植物多样性格局及多假说验证
}

\author{
欧文慧 ${ }^{1}$ 刘亚恒 1,2 李 娜 ${ }^{1}$ 徐芷妍 ${ }^{1}$ 彭秋桐 $^{1}$ 杨予静 $^{1}$ 李中强 $^{*}$
}

${ }^{1}$ 湖北大学资源环境学院, 区域开发与环境响应湖北省重点实验室, 武汉 $430062 ;{ }^{2}$ 湖北省生态环境科学研究院(省生态环境工程评估中心), 武汉 430072

摘 要 物种多样性格局同时受到多个因子和过程的综合作用。以往对水生植物多样性格局形成机制的研究主要集中在几何 限制、水分能量状况或随机过程等少数因子方面。该研究通过野外调查, 研究柴达木盆地水生植物沿经度和纬度梯度的分布 格局, 并验证了对物种多样性分布格局影响较大的水分-能量假说、栖息地异质性假说、空间自相关、物种-面积效应和中域 效应这5种假说。主要结果表明柴达木盆地水生植物多样性沿经度和纬度梯度均呈现“ $\cap$ ”形单峰格局。回归分析显示中域效 应和物种-面积效应显著影响柴达木盆地水生植物多样性格局, 而水分-能量、栖息地异质性假说及空间自相关对该区域水生 植物多样性格局影响较小。方差分解显示中域效应对柴达木盆地水生植物多样性经度和纬度格局的单独解释率分别为 68.41\%和 $66.91 \%$, 该结果表明柴达木盆地水生植物多样性格局主要受几何限制和扩散限制影响。结合以往研究结果, 该研究 进一步证实几何限制和随机效应可能是影响中国干旱区水生植物多样性分布格局的重要自然因素。

关键词 多样性格局; 中域效应; 水分-能量假说; 空间自相关; 物种-面积效应; 栖息地异质性假说; 水生植物; 柴达木盆地

欧文慧, 刘亚恒, 李娜, 徐芷妍, 彭秋桐, 杨予静, 李中强 (2021). 柴达木盆地水生植物多样性格局及多假说验证. 植物生态学报, 45, 1213-1220. DOI: 10.17521 /cjpe.2020.0364

\section{Testing multiple hypotheses for the richness pattern of macrophyte in the Qaidam Basin of Northwest China}

\author{
OU Wen-Hui ${ }^{1}$, LIU Ya-Heng ${ }^{1,2}$, LI Na $^{1}$, XU Zhi-Yan ${ }^{1}$, PENG Qiu-Tong ${ }^{1}$, YANG Yu-Jing ${ }^{1}$, and LI Zhong-Qiang ${ }^{1 *}$
}

${ }^{1}$ Faculty of Resource and Environment, Hubei University, Hubei Province Key Laboratory of Regional Development and Environmental Response, Wuhan 430062, China; and ${ }^{2}$ Hubei Academy of Environmental Sciences (Hubei Provincial Eco-Environmental Engineering Assessment Center), Wuhan 430072, China

\begin{abstract}
Aims Patterns of species richness are influenced by both ecological factors and processes. The formation mechanisms of macrophyte richness pattern have been mainly concentrated in a few aspects, such as geometric constraints, water-energy status, or random processes.

Methods In this study, using collected macrophyte species-level data in the Qaidam Basin, we examined macrophyte richness patterns along latitudinal and longitudinal gradients, and tested the mid-domain effect (MDE), area effect (AE), water-energy hypothesis, spatial autocorrelation, and habitat heterogeneity hypothesis for the geographical patterns.

Important findings Our study showed that the macrophyte richness in the Qaidam Basin showed a humpshaped pattern along latitudinal and longitudinal gradients. Regression analyses indicated that MDE and AE, rather than water-energy hypothesis, spatial autocorrelation, or habitat heterogeneity hypothesis significantly influenced macrophyte richness in the Qaidam Basin. Variation partitioning showed that the explanatory power of MDE for longitudinal and latitudinal richness patterns of macrophyte was $68.41 \%$ and $66.91 \%$, respectively. This result implies that macrophyte richness in the Qaidam Basin is mainly affected by geometric and dispersal restrictions. This study further confirms that geometric constraints and random processes may be important natural factors affecting the pattern of macrophyte richness in the arid area of Northwest China.

Key words richness pattern; mid-domain effect; water-energy hypothesis; spatial autocorrelation; species-area effect; habitat heterogeneity hypothesis; macrophyte; Qaidam Basin

Ou WH, Liu YH, Li N, Xu ZY, Peng QT, Yang YJ, Li ZQ (2021). Testing multiple hypotheses for the richness pattern of macrophyte in the Qaidam Basin of Northwest China. Chinese Journal of Plant Ecology, 45, 1213-1220. DOI: 10.17521/cjpe.2020.0364

收稿日期Received: 2020-11-09 接受日期Accepted: 2021-06-30

基金项目: 国家自然科学基金(31570366)、湖北省教育厅高等学校优秀中青年科技创新团队计划项目(T201701)和湖北自然科学基金创新群体项目 (2020CFA005)。Supported by the National Natural Science Foundation of China (31570366), the Young and Middle-aged Science \& Technology Innovation Team Project of Hubei Provincial Department of Education (T201701), and the Innovative Research Group Project of the National Natural Science Foundation of Hubei Province (2020CFA005).

* 通信作者Corresponding author (lizhq@hubu.edu.cn)
\end{abstract}


物种多样性的分布格局及形成机制一直是宏观 生态学的研究热点(Gaston, 2000)。近几十年, 许多 有关陆生植物、哺乳类和鸟类等脊椎动物的多样性 分布格局研究发现物种多样性呈现出随纬度和海拔 的升高而减少的格局(Gaston, 2000; Tognelli \& Kelt, 2004; Buckley et al., 2010; Kehoe et al., 2015), 同样, 越来越多的研究显示不同的生物类群或不同地理尺 度下物种多样性分布格局不同。中美洲的珊瑚群落 丰富度随时间推移，在小尺度范围内基本保持不变, 而在大尺度范围内则呈现显著下降的趋势; 而北美 鸟类丰富度在小尺度下基本不变, 但在较大尺度上 则表现出上升趋势(Bhattarai et al., 2004; Chase et al., 2019)。这些研究表明有关物种多样性格局及形成机 制的重要科学问题仍需要在不同尺度、不同生物类 群上进行深入研究(Hortal et al., 2015; Alahuhta \& Aroviita, 2016)。

相对陆生生态系统而言, 关于水生生态系统物 种多样性空间格局的研究相对较少。近年来, 一些 研究者开始关注不同尺度下水生生态系统中物种的 丰富度格局，例如节肢动物 (Pearson \& Boyero, 2009)、鱼类(Tisseuil et al., 2013)、两栖动物(Pearson \& Boyero, 2009; Tisseuil et al., 2013)、水生哺乳动物 和水禽(Tisseuil et al., 2013)等。关于大型水生植物 多样性格局的一些研究结果显示物种丰富度并不遵 循随纬度升高而降低的一般规律(Crow，1993), 而 是遵循单峰纬度模式, 在全球尺度上, 中低纬度的 亚热带或温带地区可能出现较高的物种丰富度 (Hutchinson, 1975; Murphy et al., 2019, 2020)。这些 研究结果表明, 不同的研究区域、空间尺度、生境 类型和地理位置上大型水生植物的物种丰富度格局 及影响因子不同(Heino, 2011; Zhang et al., 2019), 需要在不同尺度、不同气候带上来研究水生植物多 样性及形成机制。

为了解释物种多样性格局的形成机制, 近几十 年有上百种假设被生态学家检验 (Willing et al., 2003)。物种多样性格局由众多因素和过程决定, 包 括板块运动、环境变化及物种形成与进化等(Qian, 1999), 在目前多样性格局形成机制的众多假设中, 中域效应(Willig et al., 2003)、栖息地异质性假说 (Jetz \& Rahbek, 2002)、物种-面积效应(Rahbek, 1997; 唐志尧等, 2009)、水分-能量假说(Hawkins et al., 2005)和空间自相关(Gould, 1970)等假说影响最大。
最近, 水生植物多样性格局形成机制研究表明由于 水生植物多为广布种, 气候因素对其多样性格局影 响较小(Santamaría, 2002; Xu et al., 2019; 李宇辉等, 2020), 影响水生植物多样性格局的主要因子包括 几何限制(Zhang et al., 2019; Li et al., 2020; 田玉清等, 2020)、面积效应(Rolon \& Maltchik, 2006; Li et al., 2020)、水分-能量因素(Alahuhta et al., 2017)、水质 (Alahuhta et al., 2017; Elo et al., 2018)及人类影响等 (Alahuhta \& Aroviita, 2016; Elo et al., 2018)。然而, 这些对水生植物多样性格局解释的研究主要集中在 少数因子或过程等方面, 从多因子或多过程对一个 区域水生植物多样性格局形成机制的研究很少。

柴达木盆地位于中国西北干旱区, 为典型温带 荒漠气候, 其植物种类贫乏, 种类趋向于集中在少 数大科及中小型科中, 区系的优势现象明显(陈桂 琛等, 2002)。同时, 研究发现水生植物在西北干旱 区的分布呈现明显的异域性和生态隔离, 即在相互 隔离的水生环境中具有相似的水生植物群落以及物 种组成(李中强, 2005)。本研究以柴达木盆地的水生 植物为例, 研究该区域水生植物多样性沿经度、纬 度分布格局, 检验中域效应、物种-面积效应、空间 自相关、栖息地异质性假说及水分-能量假说, 以探 讨该区域水生植物多样性分布格局形成机制, 为该 地区水生植物资源及湿地生态系统的保护提供参考 依据。

\section{1 材料和方法}

\section{1 研究区概况}

柴达木盆地 $\left(34.75^{\circ}-39.33^{\circ} \mathrm{N}, 87.82^{\circ}-99.28^{\circ} \mathrm{E}\right)$ 位于青藏高原北部, 是一个由阿尔金山、祁连山、 昆仑山和布尔汗布达山环绕而形成的封闭性巨大山 间断陷内陆盆地, 其东西长约 $800 \mathrm{~km}$, 南北宽 $350 \mathrm{~km}$, 面积约 $2.5 \times 10^{5} \mathrm{~km}^{2}$ 。柴达木盆地底部海拔 2 676-3 $200 \mathrm{~m}$, 是青藏高原陷落最深的地区, 为典 型性高原盆地, 冬季寒冷干燥, 夏季温暖少雨, 日 照充足, 太阳辐射强, 蒸发强烈。盆地内河流主要来 源是周围山区的融雪水、降水。河流数量多而分散, 流程短, 流量小。河网分布不均, 东部河网密集, 西 部河网稀疏, 而中部为无径流区(吴玉虎和史惠兰, 2018)。盆地内大部分地区被戈壁和沙漠所覆盖, 地 表盐碱化严重, 植被稀疏, 旱生植物为主要组成物 种(邢星等, 2014)。 


\section{2 野外调查与数据获取}

水生植物调查于 2016年7-9月在柴达木盆地进 行, 调查水体类型包括湖泊、河流、沼泽、库塘、 水田和沟渠等; 调查采用公路沿线调查和重点区域 调查相结合的方式, 整个研究区域共设置 45 个调查 样点(图1), 每个样点采用随机方法进行取样, 根据 样点植被分布面积大小, 设置 $10 \mathrm{~m} \times 10 \mathrm{~m}$ 样方1-3 个, 共调查样方 99 个。样点水平分布范围在 $36.00^{\circ}-$ $39.33^{\circ} \mathrm{N}, 93.90^{\circ}-101.08^{\circ} \mathrm{E}$ 之间, 海拔高度范围是 2624-3 345 m。调查的水生植物包括大型水生藻 类、偋类植物亚门和种子植物亚门中光合作用器官 至少数月或永久沉没于水中或浮在水面的植物。

水生植物群落调查参照《植物群落清查的主要 内容、方法和技术规范》 (方精云等, 2009), 记录样 方中所有水生/湿生植物种类, 用手持GPS获取调查 样点经纬度和海拔; 水体环境因子数据中温度、电 导率、溶解性固体总量(TDS)由便携式水质检测仪 YSI测定, $\mathrm{pH}$ 由 $\mathrm{pH}$ 计测定, 盐度由盐度计测定, 水 深数据由水位探测仪测定。

研究区域水分和能量数据分别由区域年降水 量 $(P A N)$ 和年潜在蒸散量 $(P E T)$ 代表。PAN数据获取 自气候危害组红外线降水站(CHIRPS)网站(https: //data.chc.ucsb.edu/products/CHIRPS-2.0/, 空间分辨 率为 $\left.0.05^{\circ} \times 0.05^{\circ}\right) ; P E T$ 基础数据获取自美国蒙大拿 大学官网(http://files.ntsg.umt.edu/data/NTSG_Products/
MOD16/, 空间分辨率为1 km), 结合实测地理坐标, 通过ArcGIS 10.2的提取值到点功能, 提取每个位点 2000-2014年各年PAN及 PET数据, 计算平均值。

面积数据提取自数字高程模型(DEM), 基础数 据来源于中国科学院计算机网络信息中心国际科学 数据网站 (http://www.gscloud.cn/), 空间分辨率为 $90 \mathrm{~m} \times 90 \mathrm{~m}$ 。

\section{3 数据分析}

中域效应的验证通过中域效应分析软件 RangeModel经1 000 次Monte Carlo随机预测, 得到 95\%置信区间内的多样性指数结果作为预测值, 线 性回归分析建立预测值和调查值的关系，检验柴达 木盆地水生植物多样性格局的几何限制和随机过程 影响(Colwell, 2008)。

水分-能量假说的验证采用O'Brien $(1993 ， 1998)$ 的方法进行多元回归:

$\log S=a+b \times(\log P A N)+c \times(\log P E T)-d \times(\log P E T)^{2}$

式中, $S$ 代表物种丰富度。S、PAN和 $P E T$ 均取对数，以 满足方差齐性。

空间自相关通过Moran's I指数的计算以确定植 物物种丰富度在空间位置上的分布模式(刘吉平等, 2010)。计算公式如下:

$$
I=\frac{n \sum_{i=1}^{n} \sum_{j=1}^{n} w_{i j}\left(x_{i}-\bar{x}\right)\left(x_{j}-\bar{x}\right)}{\sum_{i=1}^{n} \sum_{j=1}^{n} w_{i j} \sum_{i=1}^{n}\left(x_{i}-\bar{x}\right)^{2}}
$$

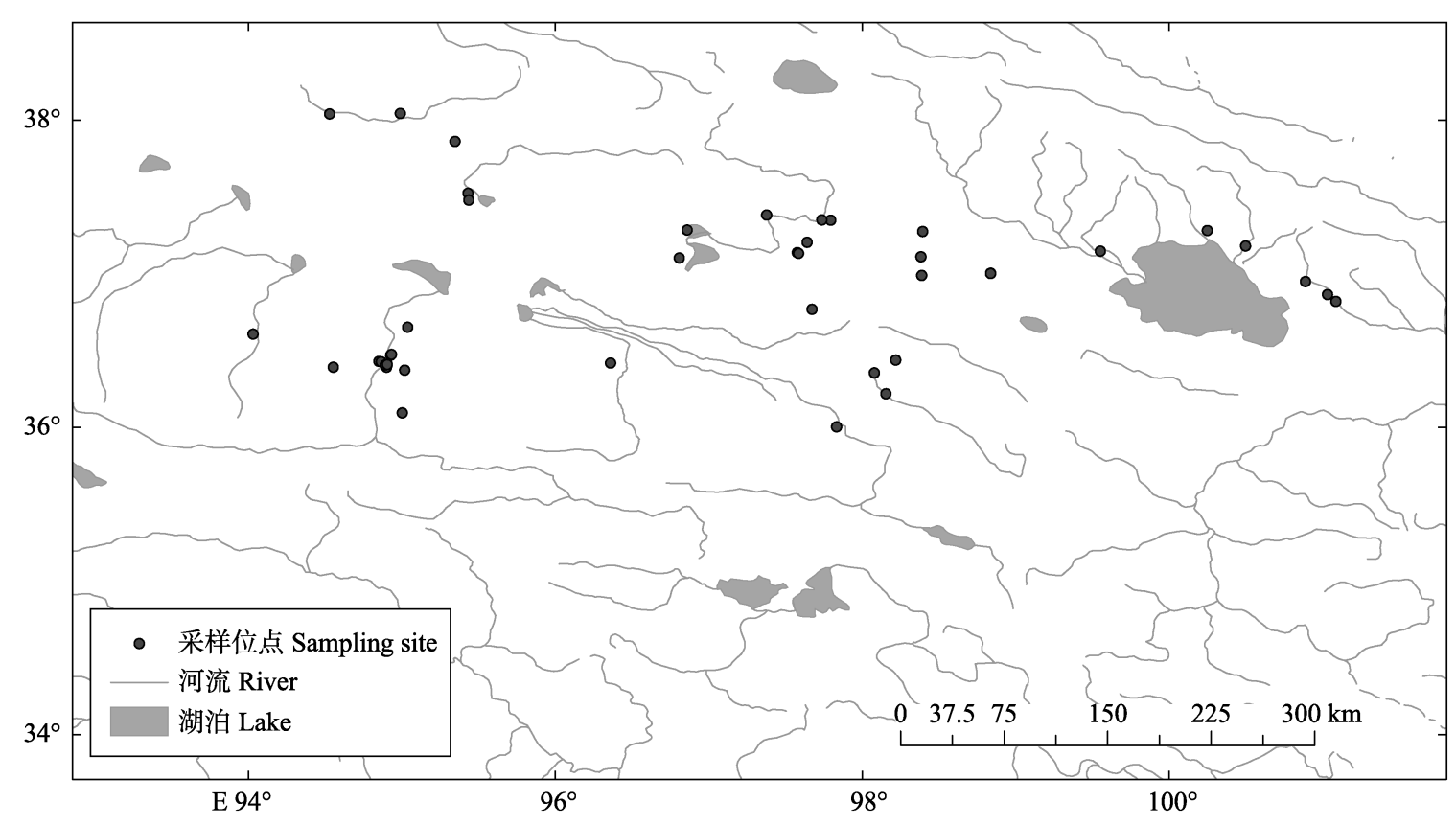

图1 柴达木盆地水生植物调查采样点分布图。

Fig. 1 A map of macrophyte survey sites in the Qaidam Basin of northwest China. 
式中, $I$ 表示全局空间自相关指数; $x_{i}$ 是变量 $X$ 在空间 位置 $i$ 上的属性值, $\bar{x}$ 为变量的均值; $n$ 为样点总个 数; $w_{i j}$ 为空间权重矩阵。 $I$ 的值域为 $(-1.0,1.0)$, 当 $I>$ 0 时, 表现为空间正相关; 当 $I<0$ 时, 表现为空间负 相关; 当I趋近于 0 时, 无空间自相关。

通过巢式取样方法按 $0.5^{\circ}$ 经度间隔, $0.2^{\circ}$ 纬度间 隔不断扩大取样面积, 通过ArcGIS 10.2软件提取研 究区DEM图中各取样范围内的3D表面积, 统计取 样范围内的物种数并绘制物种累积曲线(Gray et al., 2004), 构建柴达木盆地水生植物物种-面积效应。

栖息地异质性假说是通过对物种丰富度和水环 境数据之间进行冗余分析(RDA), 篮选具有显著相 关关系的水环境因子数据与物种丰富度数据进行回 归分析。

利用RangeModel软件进行中域效应检验; 水分能量假说验证利用 1 stOpt 1.5 软件多元线性回归进行; 全局空间自相关的Moran's I指数通过ArcGIS 10.2 软件计算得到; 物种-面积曲线在Microsoft Excel 2016中绘制; 利用Canoco 5软件进行环境因子的 $\mathrm{RDA}$; 利用R 3.5.3软件进行方差分解分析各显著影 响柴达木盆地水生植物多样性格局变量的贡献值。 文章中图分别由ArcGIS 10.2、Microsoft Excel 2016、 R 3.5.3和Adobe Photoshop CS6软件制作。

\section{2 结果}

\section{1 柴达木盆地水生植物多样性及其分布格局}

本次调查共采集到水生植物 17 科 41 属 49 种。其 中轮藻纲 1 科 1 属 1 种, 偋类植物 1 科 1 属 1 种, 被子植 物 15 科 40 属 47 种; 其中分布较广的水生植物主要包

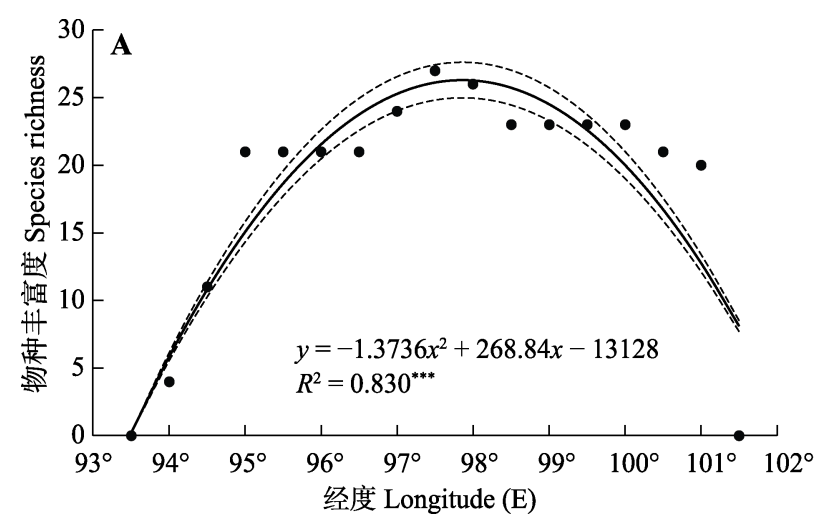

括轮藻(Chara vulgaris)、水麦冬(Triglochin palustre)、海非菜(Triglochin maritimum)、篦齿眼子菜 (Stuckenia pectinata)、北疆薹草(Carex arcatica)、牛 毛毡(Eleocharis acicularis)、芦苇(Phragmites australis)和水苶(Polygonum hydropiper)等。

柴达木盆地地区水生植物多样性在经向和纬向 上均呈现单峰型格局。经向变化上, 物种丰富度在 $97^{\circ}-98^{\circ} \mathrm{E}$ 之间达到最高值(图2A); 纬向变化上, 物 种丰富度自南向北先上升, 后下降, 在 $37.0^{\circ}-37.5^{\circ}$ $\mathrm{N}$ 之间达到最高(图2B)。

\section{2 柴达木盆地水生植物多样性格局的假说验证}

中域效应验证结果显示, 在 $95 \%$ 置信度的条件 下, 经纬度梯度调查值与对应的中域效应预测值之 间相关性均极显著 $(p<0.001)$ 。中域效应对物种多样 性格局的解释度在经度梯度上为 $83.0 \%$ (图2A); 在 纬度梯度上为 $77.1 \%$ (图2B)。

物种-面积效应分析显示柴达木盆地地区水生 植物丰富度的增幅随着面积的增加而逐渐下降, 当 面积为 $2500 \mathrm{~km}^{2}$ 时, 水生植物物种丰富度达到最高 且基本不再增加(图3)。物种丰富度与采样面积之间 呈幂函数关系, 在置信度为 $95 \%$ 的情况下, 回归分 析结果表明调查值与预测值之间呈极显著相关关系 $(p<0.001)$ 。

$\mathrm{RDA}$ 表明所检测的水环境因子中, 仅TDS与柴 达木盆地水生植物丰富度呈显著相关关系 $(p<0.05)$ (表1)。

回归分析结果显示水分-能量假说和栖息地异 质性假说与柴达木盆地水生植物丰富度相关性不显 著 $(p>0.05)$ 。空间自相关分析表明柴达木盆地水生

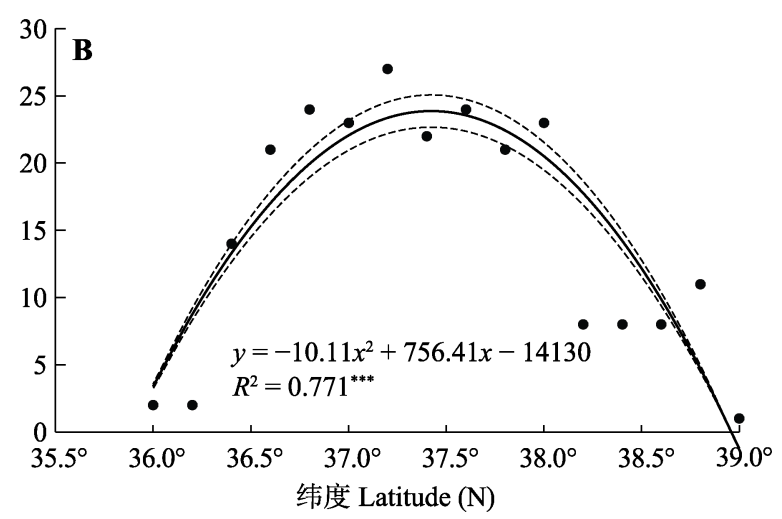

图2 柴达木盆地水生植物的经度和纬度多样性分布格局。实线为中域效应模型预测值, 虚线为 $95 \%$ 的置信区间。***, $p<$ 0.001 。

Fig. 2 Longitudinal and latitudinal pattern of macrophyte richness at the Qaidam Basin. Solid lines represent predicted richness by Range Model based on mid-domain effect. Dashed lines represent the $95 \%$ confidence interval. ${ }^{* *}, p<0.001$. 


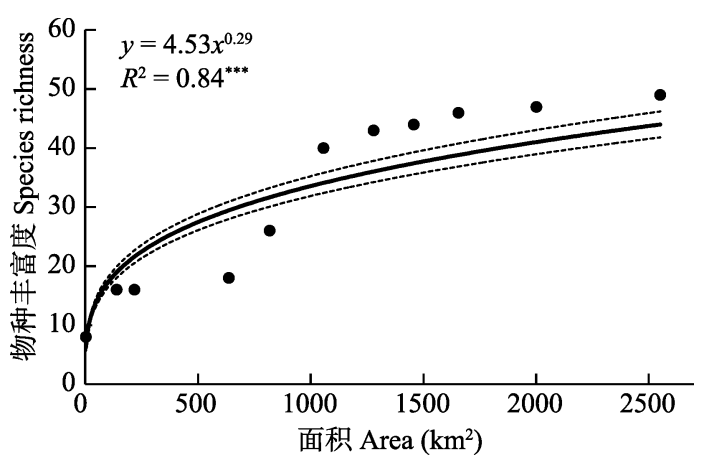

图3 柴达木盆地水生植物物种-面积关系曲线。实线为物 种-面积效应模型预测值，虚线为 $95 \%$ 的置信区间。***, $p<$ 0.001 。

Fig. 3 Species-area relationship curve of macrophytes in the Qaidam Basin. The solid line represents predicted richness by species-area effect, and dashed lines the $95 \%$ confidence interval. ${ }^{* * *}, p<0.001$.

植物物种多样性空间分布模式与随机模式无显著差 异，其Moran's $I=0.06$ (表2)。

根据前面的验证结果，对具有显著影响的中域 效应和物种-面积效应进行解释贡献率方差分解分 析。结果显示，中域效应和物种-面积效应对经度多 样性格局解释率最高, 共解释其变量的 $94.01 \%$, 其 中，中域效应和物种-面积效应单独解释率分别为
表1 水环境因子对柴达木盆地水生植物丰富度的解释度

Table 1 Explanatory power of aquatic environmental factors on macrophyte species richness in the Qaidam Basin

\begin{tabular}{lcc}
\hline $\begin{array}{l}\text { 水环境因子 } \\
\text { Aquatic environment factor }\end{array}$ & $\begin{array}{c}\text { 解释度 } \\
\text { Explain (\%) }\end{array}$ & $p$ \\
\hline 溶解性固体总量 TDS & 2.0 & $0.046^{*}$ \\
盐度 Salinity & 1.9 & 0.064 \\
$\mathrm{pH}$ & 1.5 & 0.154 \\
电导率 Conductivity & 1.3 & 0.250 \\
水深 Water depth & 1.0 & 0.460 \\
温度 Temperature & 0.9 & 0.478 \\
\hline
\end{tabular}

TDS, total dissolved solids. *, $p<0.05$.

$68.41 \%$ 和 $25.60 \%$, 二者共同解释率为0 (图4A); 相对 水生植物多样性经度格局而言，两种假说对物种多 样性纬度梯度格局解释率较低，共解释了变量的 $68.16 \%$ ，未解释率达 $31.84 \%$; 中域效应对多样性格 局的解释率较高, 其单独解释率达 $66.91 \%$, 物种-面 积效应对纬度格局解释率为 0 , 二者共同解释率为 $1.25 \%$ (图4B)。

\section{3 讨论}

由于不具备陆地和海洋生境所具有的连续性特

表2 柴达木盆地水生植物多样性在水分-能量假说、空间自相关和栖息地异质性假说下的回归方程

Table 2 Regression equations of macrophyte richness in the Qaidam Basin under hypotheses of water-energy, spatial autocorrelation, and environmental heterogeneity

\begin{tabular}{llc}
\hline 假说 Hypothesis & 回归方程 Regression equation & $p$ \\
\hline 水分-能量假说 Water-energy hypothesis & $\log S=1.82-0.01 \log P A N-0.20 \log P E T-0.02 \log P E T^{2}$ & 0.051 \\
空间自相关 Spatial autocorrelation & - & 0.250 \\
栖息地异质性假说 Habitat heterogeneity hypothesis & $S=6.48-0.49 T D S$ & 0.330 \\
\hline
\end{tabular}

$P A N$, 年降水量; $P E T$, 潜在蒸散量; $S$, 物种数; $T D S$, 溶解性固体总量。

$P A N$, annual precipitation; $P E T$, potential evapotranspiration; $S$, species; TDS, total dissolved solids.

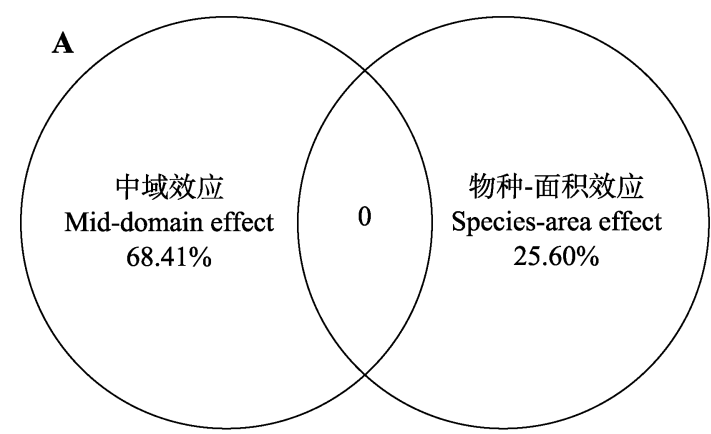

残差 Residuals $=5.99 \%$

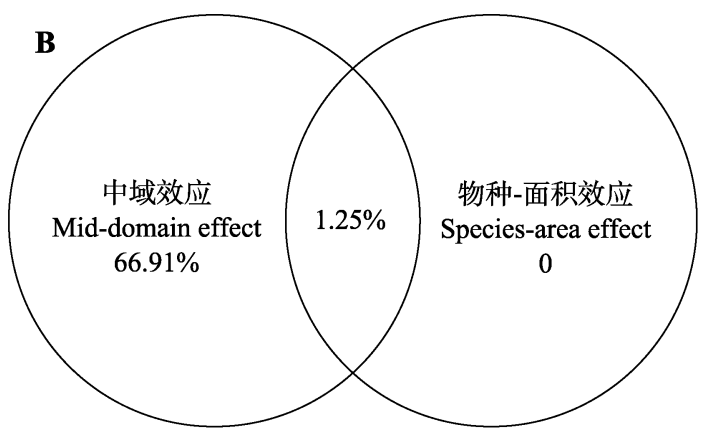

残差 Residuals $=31.84 \%$

图4 中域效应及物种-面积效应对柴达木盆地水生植物经度和纬度多样性格局的相对影响。A, B分别为经度和纬度的中域 效应和物种-面积效应的方差分解韦恩图。负值以 0 表示, 代表无解释度。

Fig. 4 Relative explanatory power of mid-domain effect and species-area effect on longitudinal and latitudinal patterns of macrophyte richness in the Qaidam Basin. A, B are the Venn diagram of the variance partitioning of mid-domain effect and species-area effect on longitude and latitude, respectively. The negative value is represented by 0 , which means lack of explanatory power. 
征, 淡水生物类群在较大尺度上所呈现的物种多样 性格局相对复杂(Heino, 2011; Alahuhta et al., 2017; Xu et al., 2019)。早先研究表明水生植物多样性分布 格局因空间范围和地理位置的变化而不同(Heino \& Toivonen, 2008; Heino, 2011; Alahuhta et al., 2017; Xu et al., 2019; 田玉清等, 2020)。与同一气候区河 西走廊水生植物多样性格局研究结果(田玉清等, 2020)相似, 柴达木盆地水生植物物种多样性沿水 平方向的纬度和经度梯度上呈现出明显的单峰分布 模式。单峰分布模式可能是较为普遍的物种多样性 格局(Lomolino, 2001; Rahbek, 2005)。最近研究显示 一些陆生植物水平梯度多样性格局也呈现类似的单 峰分布模式(Wang et al., 2011; Shrestha et al., 2018)。 有趣的是, 与该区域水生植物多样性格局单峰分布 模式恰恰相反, 该区域陆生植物多样性呈现U形的 分布格局(王健铭等, 2017)。其可能的解释是陆生和 水生生态系统中物种多样性格局形成的驱动因素不 同，如中国西北干旱区，与水相关因子如年降水量 是决定陆生植物多样分布格局的主要因素(王健铭 等, 2017); 而水分对水生植物分布影响可能较小。

在 5 个被检验的假说中，与邻近区域河西走廊 水生植物多样性研究结果(田玉清等, 2020)一致, 我 们的研究结果清楚证明中域效应是影响柴达木盆地 水生植物多样性格局形成的主要机制。中域效应认 为物种的分布范围和分布中心在有边界限制的区域 内是随机的, 不同物种分布的范围在边界的限制下, 中心区域的重叠程度大于边界附近, 形成中间高于 边缘的物种多样性单峰格局(Colwell, 2008)。已有的 许多较大尺度上有关水生植物多样性的研究表明, 物种扩散的边界限制普遍存在(Capers et al., 2010; Mikulyuk et al., 2011; Alahuhta \& Heino, 2013; Viana et al., 2014)。柴达木盆地位于青藏高原北部, 被众 多山脉环绕, 盆地内部沙漠戈壁遍布, 使得其中的 水生生境形成了边界清晰且相对孤立的水体, 水生 植物扩散受到限制。有趣的是, 尽管物种-面积效应 显著影响柴达木盆地水生植物多样性格局的形成, 但其对该区域水生植物多样性经度格局的影响远大 于对水生植物纬度格局的影响, 其原因需要进一步 研究。同样, 最近关于整个中国西部干旱区水生植 物多样性格局的研究(Li et al., 2020)表明, 其多样 性格局主要受中域效应和物种-面积效应共同影响, 而本研究表明中域效应和物种-面积效应对柴达木
盆地水生植物多样性格局共同解释率极低, 其可能 原因是尺度不同，影响物种多样性格局的因素不同 (Shmida \& Wilson, 1985; Michael et al., 2007)。

相对于中域效应和物种-面积效应，水分-能量 假说、栖息地异质性假说和空间自相关对柴达木盆 地水生植物多样性格局影响较小, 这可能与水环境 特征和水生植物特性相关。相对于陆生生境，水环 境/湿地环境中水分相对充足; 同时水生植物多为 广布种，生态幅较宽，适应性强(Santamaría，2002), 因此水分-能量和栖息地异质性假说对柴达木盆地 水生植物多样性格局影响较小。同时柴达木盆地区 域, 水生生境较少、分布间隔较远且分散, 相邻样点 之间的物种多样性与其空间位置之间的联系较弱, 近似呈现随机分布模式，空间自相关对多样性格局 影响较少。

\section{4 结论}

本次调查在柴达木盆地共采集到水生植物 17 科 41属49种。该区域水生植物多样性沿经度、纬度梯

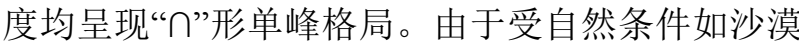
和高山阻挡，高度独立的水生生境和水生植物自身 属性如分布广泛、生态幅宽等影响, 柴达木盆地水 生植物多样性格局主要受中域效应影响; 而水分-能 量动态、栖息地异质性假说及空间自相关对该区域 多样性格局影响较小。结合以往研究结果(Li et al., 2020; 田玉清等, 2020), 本研究进一步证明几何限 制和随机效应可能是影响中国干旱区水生植物多样 性分布格局的重要因素。

\section{参考文献}

Alahuhta J, Aroviita J (2016). Quantifying the relative importance of natural variables, human disturbance and spatial processes in ecological status indicators of boreal lakes. Ecological Indicators, 63, 240-248.

Alahuhta J, Ecke F, Johnson LB, Sass L, Heino J (2017). A comparative analysis reveals little evidence for niche conservatism in aquatic macrophytes among four areas on two continents. Oikos, 126, 136-148.

Alahuhta J, Heino J (2013). Spatial extent, regional specificity and metacommunity structuring in lake macrophytes. Journal of Biogeography, 40, 1572-1582.

Bhattarai KR, Vetaas OR, Grytnes JA (2004). Fern species richness along a central Himalayan elevational gradient, Nepal. Journal of Biogeography, 31, 389-400.

Buckley HL, Miller TE, Ellison AM, Gotelli NJ (2010). 
Reverse latitudinal trends in species richness of pitcherplant food webs. Ecology Letters, 6, 825-829.

Capers RS, Selsky R, Bugbee GJ (2010). The relative importance of local conditions and regional processes in structuring aquatic plant communities. Freshwater Biology, 55, 952-966.

Chase JM, McGill BJ, Thompson PL, Antão LH, Bates AE, Blowes SA, Dornelas M, Gonzalez A, Magurran AE, Supp SR, Winter M, Bjorkman AD, Bruelheide H, Byrnes JEK, Cabral JS, et al. (2019). Species richness change across spatial scales. Oikos, 128, 1079-1091.

Chen GC, Huang ZW, Lu XF, Peng M (2002). Characteristics of wetland and its conservation in the Qinghai Plateau. Journal of Glaciology and Geocryology, 24, 254-259. [陈 桂琛, 黄志伟, 卢学峰, 彭敏 (2002). 青海高原湿地特 征及其保护. 冰川冻土, 24, 254-259.]

Colwell RK (2008). RangeModel: tools for exploring and assessing geometric constraints on species richness (the mid-domain effect) along transects. Ecography, 31, 4-7.

Crow GE (1993). Species diversity in aquatic angiosperms: latitudinal patterns. Aquatic Botany, 44, 229-258.

Elo M, Alahuhta J, Kanninen A, Meissner KK, Seppälä K, Mönkkönen M (2018). Environmental characteristics and anthropogenic impact jointly modify aquatic macrophyte species diversity. Frontiers in Plant Science, 9, 1001. DOI: $10.3389 /$ fpls.2018.01001.

Fang JY, Wang XP, Shen ZH, Tang ZY, He JS, Yu D, Jiang Y, Wang ZH, Zheng CY, Zhu JL, Guo ZD (2009). Methods and protocols for plant community inventory. Biodiversity Science, 17, 533-548. [方精云, 王襄平, 沈泽昊, 唐志 尧, 贺金生, 于丹, 江源, 王志恒, 郑成洋, 朱江玲, 郭 兆迪 (2009). 植物群落清查的主要内容、方法和技术规 范. 生物多样性, 17, 533-548.]

Gaston KJ (2000). Global patterns in biodiversity. Nature, 405, 220-227.

Gould P (1970). Is Statistix Inferens the geographical name for a wild goose? Economic Geography, 46, 439-448.

Gray JS, Ugland KI, Lambshead J (2004). On species accumulation and species-area curves. Global Ecology and Biogeography, 13, 567-568.

Hawkins BA, Diniz-Filho JAF, Weis AE (2005). The middomain effect and diversity gradients: Is there anything to learn? The American Naturalist, 166, 140-143.

Heino J (2011). A macroecological perspective of diversity patterns in the freshwater realm. Freshwater Biology, 56, 1703-1722.

Heino J, Toivonen H (2008). Aquatic plant biodiversity at high latitudes: patterns of richness and rarity in Finnish freshwater macrophytes. Boreal Environment Research, 13, 114.

Hortal J, Bello F, Diniz-Filho J, Lewinsohn T, Lobo J, Ladle RJ (2015). Seven shortfalls that beset large-scale knowledge of biodiversity. Annual Review of Ecology, Evolution, and Systematics, 46, 523-549.

Hutchinson GE (1975). A Treatise on Limnology. III. Limnological Botany. John Wiley, New York.

Jetz W, Rahbek C (2002). Geographic range size and determinants of avian species richness. Science, 297, 1548-1551.

Kehoe L, Kuemmerle T, Meyer C, Levers C, Václavík T, Kreft $\mathrm{H}$ (2015). Global patterns of agricultural land-use intensity and vertebrate diversity. Diversity and Distributions, 21, 1308-1318.

Li YH, Hao T, Gong XS, Yang YJ, Li ZQ (2020). Species richness and influencing factors of aquatic plant in the Kaidu River Basin, Xinjiang, China. Chinese Journal of Applied Ecology, 31, 1691-1698. [李宇辉, 郝涛, 龚旭昇, 杨予静, 李中强 (2020). 新疆开都河流域水生植物多样 性及其影响因素. 应用生态学报, 31, 1691-1698.]

Li ZQ (2005). Study on the Biodiversity of Aquatic Plants in the Arid Zone of Northwest China. PhD dissertation, Wuhan University, Wuhan. [李中强 (2005). 中国西北干旱区水 生植物多样性研究. 博士学位论文, 武汉大学, 武汉.]

Li ZQ, Tian YQ, Zhou YL, Gong XS, Peng QT, Xu ZY, Cai YJ (2020). Geometric constraints and area effects shape geographical patterns of aquatic plant diversity in a highly arid region. Ecological Indicators, 116, 106502. DOI: 10.1016/j.ecolind.2020.106502.

Liu JP, Lv XG, Liu QF, Gao JQ (2010). Spatial autocorrelation analysis of wetland bird diversity in Bielahong Basin. Acta Ecologica Sinica, 30, 2647-2655. [刘吉平, 吕宪国, 刘庆 凤, 高俊琴 (2010). 别拉洪河流域湿地鸟类丰富度的空 间自相关分析. 生态学报, 30, 647-2655.]

Lomolino MV (2001). Elevation gradients of species-density: historical and prospective views. Global Ecology and Biogeography, 10, 3-13.

Michael Anderson T, Metzger KL, McNaughton SJ (2007). Multi-scale analysis of plant species richness in Serengeti grasslands. Journal of Biogeography, 34, 313-323.

Mikulyuk A, Sharma S, van Egeren S, Erdmann E, Nault ME, Hauxwell J (2011). The relative role of environmental, spatial, and land-use patterns in explaining aquatic macrophyte community composition. Canadian Journal of Fisheries and Aquatic Sciences, 68, 1778-1789.

Murphy K, Carvalho P, Efremov A, Grimaldo JT, MolinaNavarro E, Davidson TA, Thomaz SM (2020). Latitudinal variation in global range-size of aquatic macrophyte species shows evidence for a Rapoport effect. Freshwater Biology, 65, 1622-1640.

Murphy K, Efremov A, Davidson TA, Molina-Navarro E, Fidanza K, Betiol TCC, Chambers P, Grimaldo JT, Martins SV, Springuel I, Kennedy M, Mormul RP, Dibble E, Hofstra D, Lukács BA, et al. (2019). World distribution, diversity and endemism of aquatic macrophytes. Aquatic Botany, 158, 103127. DOI: 10.1016/j.aquabot.2019.06.006. 
O’Brien EM (1993). Climatic gradients in woody plant species richness: towards an explanation based on an analysis of southern Africa's woody flora. Journal of Biogeography, 20, 181-198.

O’Brien EM (1998). Water-energy dynamics, climate, and prediction of woody plant species richness: an interim general model. Journal of Biogeography, 25, 379-398.

Pearson RG, Boyero L (2009). Gradients in regional diversity of freshwater taxa. Journal of the North American Benthological Society, 28, 504-514.

Qian H (1999). Spatial pattern of vascular plant diversity in North America north of Mexico and its floristic relationship with Eurasia. Annals of Botany, 83, 271-283.

Rahbek C (1997). The relationship among area, elevation, and regional species richness in neotropical birds. The American Naturalist, 149, 875-902.

Rahbek C (2005). The role of spatial scale and the perception of large-scale species-richness patterns. Ecology Letters, 8, 224-239.

Rolon AS, Maltchik L (2006). Environmental factors as predictors of aquatic macrophyte richness and composition in wetlands of southern Brazil. Hydrobiologia, 556, 221-231.

Santamaría L (2002). Why are most aquatic plants widely distributed? Dispersal, clonal growth and small-scale heterogeneity in a stressful environment. Acta Oecologica, 23(3), 137-154.

Shmida A, Wilson MV (1985). Biological determinants of species diversity. Journal of Biogeography, 12, 1-20.

Shrestha N, Su X, Xu X, Wang Z (2018). The drivers of high Rhododendron diversity in south-west China: Does seasonality matter? Journal of Biogeography, 45, 438-447.

Tang ZY, Qiao XJ, Fang JY (2009). Species-area relationship in biological communities. Biodiversity Science, 17, 549559. [唐志尧, 乔秀娟, 方精云 (2009). 生物群落的种面积关系. 生物多样性, 17, 549-559.]

Tian YQ, Shi DL, Zhang SQ, Gong XS, Wang ZX, Li ZQ (2020). Biogeographic pattern, main community types, and the influencing factors of aquatic macrophytes in the Hexi Corridor of Northwest China. Acta Ecologica Sinica, 40, 202-212. [田玉清, 石道良, 张淑倩, 龚旭昇, 汪正祥, 李中强 (2020). 河西走廊水生植物多样性格局、群落特 征及影响因素. 生态学报, 40, 202-212.]

Tisseuil C, Cornu JF, Beauchard O, Brosse S, Darwall W, Holland R, Hugueny B, Tedesco PA, Oberdorff T (2013). Global diversity patterns and cross-taxa convergence in freshwater systems. Journal of Animal Ecology, 82, 365376.
Tognelli MF, Kelt DA (2004). Analysis of determinants of mammalian species richness in South America using spatial autoregressive models. Ecography, 27, 427-436.

Viana DS, Santamaría L, Schwenk K, Manca M, Hobæk A, Mjelde M, Preston CD, Gornall RJ, Croft JM, King RA, Green AJ, Figuerola J (2014). Environment and biogeography drive aquatic plant and cladoceran species richness across Europe. Freshwater Biology, 59, 2096-2106.

Wang JM, Wang WJ, Li JW, Feng YM, Wu B, Lu Q (2017). Biogeographic patterns and environmental interpretation of plant species richness in desert regions of Northwest China. Biodiversity Science, 25, 1192-1201. [王健铭, 王 文娟，李景文，冯益明，吴波，卢琦 (2017). 中国西北 荒漠区植物物种丰富度分布格局及其环境解释. 生物 多样性, 25, 1192-1201.]

Wang ZH, Fang JY, Tang ZY, Lin X (2011). Patterns, determinants and models of woody plant diversity in China. Proceedings of the Royal Society B: Biological Sciences, 278, 2122-2132.

Willig MR, Kaufman DM, Stevens RD (2003). Latitudinal gradients of biodiversity: pattern, process, scale, and synthesis. Annual Review of Ecology, Evolution, and Systematics, 34, 273-309.

$\mathrm{Wu}$ YH, Shi HL (2018). The seed plant flora of the Qaidam Basin and its contiguous mountainous region in Qinghai Province. Acta Botanica Boreali-Occidentalia Sinica, 38, 1542-1552. [吴玉虎, 史惠兰 (2018). 柴达木盆地及其 毗邻山地植物区系研究. 西北植物学报, 38, 1542-1552.]

Xing X, Chen H, Zhu JJ, Chen TT (2014). Water sources of five dominant desert plant species in Nuomuhong area of Qaidam Basin. Acta Ecologica Sinica, 34, 6277-6286. [邢 星, 陈辉, 朱建佳, 陈同同 (2014). 柴达木盆地诺木洪 地区5种优势荒漠植物水分来源. 生态学报，34，62776286.]

Xu J, Molinos JG, Su G, Matsuzaki SS, Akasaka M, Zhang H, Heino J (2019). Cross-taxon congruence of multiple diversity facets of freshwater assemblages is determined by large-scale processes across China. Freshwater Biology, 64, 1492-1503.

Zhang M, Molinos JG, Su G, Zhang H, Xu J (2019). Spatially structured environmental variation plays a prominent role on the biodiversity of freshwater macrophytes across China. Frontiers in Plant Science, 10, 161. DOI: 10.3389/fpls.2019.00161. 